\title{
Ventilator-Integrated Jet Nebulization Systems: Tidal Volume Control and Efficiency of Synchronization
}

\author{
Stephan Ehrmann MD PhD, Aissam Lyazidi PhD, Bruno Louis PhD, Daniel Isabey PhD, \\ Déborah Le Pennec, Laurent Brochard MD PhD, and Gabriela Apiou-Sbirlea PhD
}

\begin{abstract}
BACKGROUND: Jet nebulizers constitute the aerosolization devices most frequently used during mechanical ventilation. Continuous nebulization can influence the delivered tidal volume $\left(V_{T}\right)$ and lead to significant medication loss during expiration. Ventilators thus provide integrated jet nebulization systems that are synchronized during inspiration and ostensibly keep $V_{T}$ constant. METHODS: This was a bench study of systems integrated in the Evita XL, Avea, Galileo, and G5 ventilators. The $V_{T}$ delivered with and without nebulization, the inspiratory synchronization of nebulization, and the aerosol deposition were measured with 2 locations of the nebulizer. RESULTS: Changes in $V_{T}$ with the nebulizer were below $20 \mathrm{~mL}$ and below $10 \%$ of set $V_{T}$ for all ventilators. Synchronization was good at the beginning of insufflation, but prolonged nebulization was observed with all ventilators at the end of insufflation, until up to $1 \mathrm{~s}$ during expiration: 5-80\% of nebulization occurred during expiration with significant aerosol loss in the expiratory limb. Synchrony could be improved by (1) reducing gas compression/decompression phenomena proximal to the jet nebulizer and (2) increasing inspiratory time, which reduced the amount of nebulization occurring during expiration. Placing the nebulizer upstream in the inspiratory limb did not affect inspiratory synchrony but allowed reduction of the amount of aerosol lost in the expiratory limb. CONCLUSIONS: Jet nebulizer systems integrated in the tested ventilators are reliable in terms of $\mathrm{V}_{\mathrm{T}}$ control. Gas compression in tubing driving gas to the nebulizer delays synchronization and reduces nebulization yield if the nebulizer is placed close to the Y-piece. Increasing inspiratory time with no end-inspiratory pause reduces the expiratory loss of medication if placement of the nebulizer upstream in the inspiratory limb is not feasible. Key words: administration, inhalation [MeSH]; respiration, artificial [MeSH]; nebulizers and vaporizers [MeSH]. [Respir Care 2014;59(10):1508-1516. (C) 2014 Daedalus Enterprises]
\end{abstract}

\section{Introduction}

Jet nebulizers are simple cost-effective devices for aerosolized medication delivery. ${ }^{1}$ They are designed for single

Drs Ehrmann, Louis, Isabey, Brochard, and Apiou-Sbirlea are affiliated with the Institut National de la Santé et de la Recherche Médicale, U955, Équipe Biomécanique Cellulaire et Respiratoire, and the Centre National de la Recherche Scientifique, équipe de recherche labellisée 7240, Créteil, France. Drs Ehrmann, Louis, Isabey, and Apiou-Sbirlea are also affiliated with the Université Paris-Est Créteil, Unité Mixte de Recherche S955, Créteil, France. Dr Ehrmann is also affiliated with the Service de Réanimation Polyvalente, Centre Hospitalier Régional Universitaire de Tours, Tours, France. Dr Lyazidi is affiliated with the Service des Soins Intensifs, Hôpitaux Universitaires de Genève, Geneva, Switzerland and Institut supérieur des sciences de la santé, Université Hassan ler, Settat, Morocco. Ms Le Pennec and Dr Ehrmann are affiliated with the use, thus obviating sterilization procedures and contamination-associated risks. ${ }^{2}$ Their operation requires a 6-8 L/min gas flow; the nebulizer's internal resistance (pinhole narrowing) induces a pressure drop within the device, resulting in aerosolization through a Bernoulli ef-

\footnotetext{
Centre d'Étude des Pathologies Respiratoires, Institut National de la Santé et de la Recherche Médicale, UMR 1100/EA6305 F-37032 Tours, France, and the Faculté de Médecine de Tours, UMR 1100 Université François Rabelais, F-37032 Tours, France. Dr Brochard is also affiliated with Department of Critical Care, St Michael's Hospital, the Keenan Research Centre and the Interdepartmental Division of Critical Care Medicine, University of Toronto, Toronto, Canada. Dr Apiou-Sbirlea is currently affiliated with the Wellman Center for Photomedicine, Massachusetts General Hospital, and the Department of Dermatology, Harvard Medical School, Boston, Massachusetts.
} 


\section{Ventilator-Integrated Jet Nebulization Systems}

fect. Recent data indicate that jet nebulizers are the most common devices used for nebulization during mechanical ventilation in ICUs. ${ }^{3}$

During mechanical ventilation, the gas flow operating the jet nebulizer may interfere with the gas flow delivered by the ventilator. ${ }^{4}$ For instance, in the case of a ventilator set to deliver a $40 \mathrm{~L} / \mathrm{min}$ square waveform flow and a jet nebulizer operated with an $8 \mathrm{~L} / \mathrm{min}$ external gas source, a $20 \%$ increase in tidal volume $\left(\mathrm{V}_{\mathrm{T}}\right)$ may approximately be expected. As manual corrections of ventilator settings to take into account this extra gas flow are imprecise and cumbersome, manufacturers have implemented integrated jet nebulization systems into ventilators. ${ }^{4}$ Those systems comprise a jet nebulization port on the ventilator, providing the nebulizer's driving gas in parallel to the gas delivered through the inspiratory limb. ${ }^{5}$ This theoretically enables an automatic compensation of the insufflated volume to potentially maintain a constant $\mathrm{V}_{\mathrm{T}}$ and patient ventilation (Fig. 1). In addition to controlling $\mathrm{V}_{\mathrm{T}}$, jet nebulization systems integrated into ventilators aim to synchronize nebulization with inspiration to avoid loss of medication aerosolized during expiration. ${ }^{6}$ Thus, ventilators aim to deliver the driving gas to the nebulizer only during inspiration. ${ }^{7}$ A study from 1993 evaluated the nebulizers of a preceding generation of ventilators and showed that some performed poorly. ${ }^{5}$ To the best of our knowledge, the possible benefits of the integrated jet nebulization systems, that is, control of $\mathrm{V}_{\mathrm{T}}$ and inspiratory synchronized aerosol delivery, have not been independently evaluated despite their frequent use in ICUs and regulatory approval. ${ }^{3}$

The aim of this bench study was to measure changes in $\mathrm{V}_{\mathrm{T}}$ and to assess inspiratory synchronization when operating ventilator-integrated jet nebulization systems.

Drs Ehrmann, Louis, Isabey, Brochard, and Apiou-Sbirlea were supported in part by Grant ANR-2010 BLAN 111905 from the Agence Nationale de la Recherche. Dr Ehrmann was provided with equipment for research purposes from La Diffusion Technique Française, Penn-Century, and Dräger. Dr Brochard's laboratory received research grants from Covidien, Dräger, Vygon, Philips Respironics, and GE Healthcare; none were related to this particular study. The other authors have disclosed no conflicts of interests.

Drs Ehrmann, Lyazidi, Brochard, and Apiou-Sbirlea presented a version of this paper at the 40th Congrès International de la Société de Réanimation de Langue Française, held January 18-20, 2012, in Paris, France, and at the American Thoracic Society 2012 International Conference, held May 18-23, 2012, in San Francisco, California.

Correspondence: Stephan Ehrmann MD PhD, Service de Réanimation Polyvalente, CHRU de Tours, F37044 Tours, France. E-mail: stephane hrmann@gmail.com.

DOI: $10.4187 /$ respcare.02637

\section{QUICK LOOK}

\section{Current knowledge}

Jet nebulizers are the most commonly used devices for delivery of aerosolized medications during mechanical ventilation. Continuous nebulization augments tidal volume $\left(\mathrm{V}_{\mathrm{T}}\right)$ and may induce some loss of medication. Ventilators may incorporate nebulizer drive systems to synchronize aerosol delivery with inspiration, maintaining constant $\mathrm{V}_{\mathrm{T}}$ and avoiding drug waste.

\section{What this paper contributes to our knowledge}

Jet nebulization systems integrated in ventilators provide control of $\mathrm{V}_{\mathrm{T}}$ and may be preferred to external gas sources or empirical ventilator setting changes. Placement of the nebulizer upstream in the inspiratory limb reduces the loss of medication in the expiratory limb due to nebulization during expiration.

\section{Methods}

Jet nebulization systems integrated in 4 ICU ventilators (Evita XL [Dräger, Lübeck, Germany], Avea [CareFusion, San Diego, California], and Galileo and G5 [Hamilton Medical, Reno, Nevada]) were evaluated in a bench model of mechanical ventilation (Fig. 1). Gas flow was measured twice (Fleisch No. 2 pneumotachograph, cleaned and dried between each experiment): first, immediately distal to the nebulizer, and second, distal to the Y-piece to measure the total flow entering the lungs. The airway pressure was measured at the Y-piece. The lung model (Training and Test Lung, Michigan Instruments, Grand Rapids, Michigan) compliance and resistance were set at $60 \mathrm{~mL} / \mathrm{cm} \mathrm{H}_{2} \mathrm{O}$ and $5 \mathrm{~cm} \mathrm{H}_{2} \mathrm{O} / \mathrm{L} / \mathrm{s}$, respectively. The delivered $\mathrm{V}_{\mathrm{T}}$ was measured through flow integration over time distal to the Y-piece. Inspiratory synchronization of nebulization was evaluated measuring the time lapse between beginning of insufflation and beginning of nebulization (beginning of insufflation synchrony: see $\Delta 1$ in Fig. 1) and the time lapse between end of insufflation and end of nebulization (end of insufflation synchrony: see $\Delta 2$ in Fig. 1). The beginning and end of insufflation and nebulization were determined based on flow and pressure tracings. Nine different volume controlled ventilator settings were evaluated varying $\mathrm{V}_{\mathrm{T}}(300,500$, and $700 \mathrm{~mL})$ and square waveform inspiratory flow $(30,50$, and $70 \mathrm{~L} / \mathrm{min})$ without an end-inspiratory pause. Breathing frequency was set at 12 breaths/min, $\mathrm{F}_{\mathrm{IO}_{2}}$ at $21 \%$, and end-expiratory pressure at $5 \mathrm{~cm} \mathrm{H}_{2} \mathrm{O}$. All measurements were carried out at ambient temperature and pressure in dry conditions.

First, measurements were carried out with a standard jet nebulizer commonly used in the ICU (Micro Mist, Hudson 


\section{Ventilator-Integrated Jet Nebulization Systems}

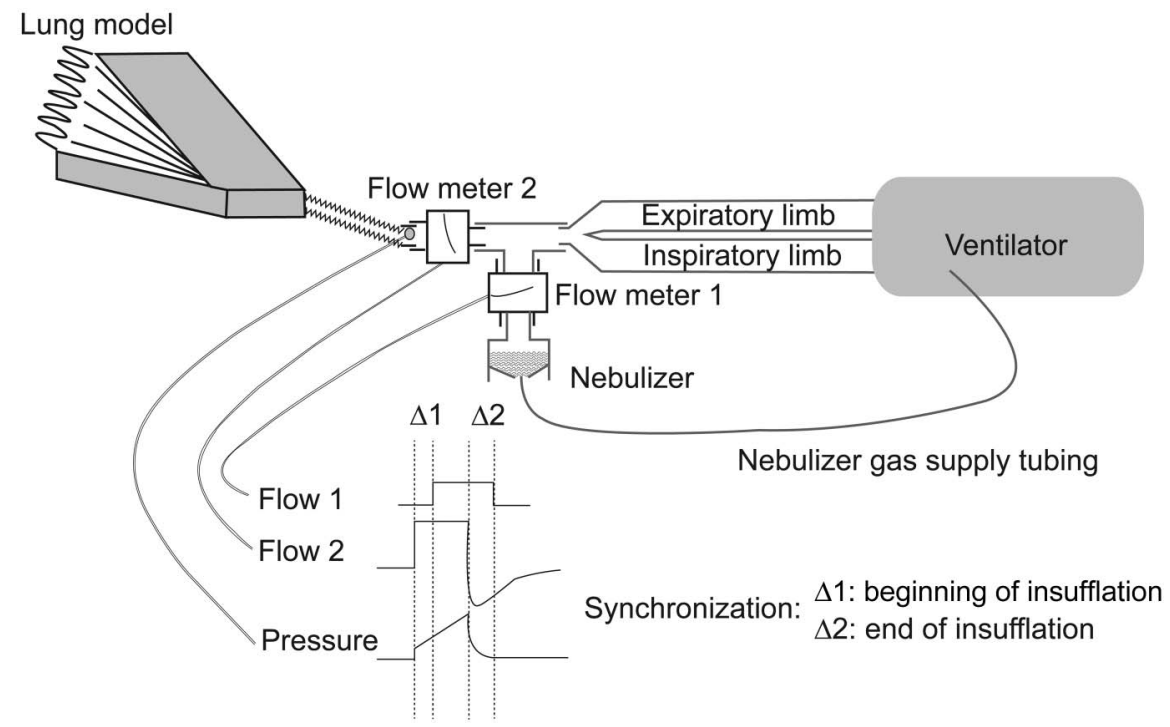

Fig. 1. $\Delta 1$ and $\Delta 2$ time lapses were computed as positive values if flow 1 started after flow 2 and negatively if otherwise. When performing tests without a nebulizer in place, the nebulizer gas supply tubing was directly connected to flow meter 1 . This tubing was cut to $2 \mathrm{~cm}$ in length to perform measurements with reduced volume of compressible gas proximal to the nebulizer.

RCI, Durham, North Carolina) filled with $0.9 \%$ sodium chloride and placed close to the Y-piece (Fig. 1). Second, to gain insight into the mechanisms ruling inspiratory synchrony/asynchrony of nebulization, measurements were also performed (1) without a nebulizer placed in the circuit; (2) with a nebulizer exhibiting a high internal resistance (Heart, WestMed, Tucson, Arizona); and (3) with the standard nebulizer but reducing the length of the nebulizer gas supply tubing to $2 \mathrm{~cm}$ (to reduce the volume of compressible gas), placing the nebulizer very close to the output of the ventilator. Indeed, we reasoned that the long tubing, driving gas from the ventilator to the nebulizer, was a source of gas compression that would continue to drive the aerosol after the end of the ventilator's insufflation. The Evita XL ventilator was also tested with nebulizer 8412935 (Dräger), as recommended by the ventilator manufacturer.

To distinguish between nebulization synchronized with insufflation as opposed to inspiration (insufflation + endinspiratory pause), all measurements were repeated with a 1-s end-inspiratory pause.

Finally, to illustrate the link between inspiratory synchronization of nebulization and aerosol delivery (and conversely, aerosol loss in the expiratory limb), deposition experiments were performed using $0.9 \%$ sodium fluoride as a tracer as previously described. ${ }^{8,9}$ Briefly, filters were placed between the endotracheal tube and the lung model (to measure delivered aerosol) and between the Y-piece and expiratory limb (to quantify aerosol loss in the expiratory limb); the Micro Mist nebulizer (filled with $4 \mathrm{~mL}$ of $0.9 \%$ sodium fluoride) was run until no more nebulization could be visually observed, and filters were dismounted
Table 1. Absolute Changes in Flow, Pressure, and Volume Delivered at the Y-piece

\begin{tabular}{lcccc}
\hline \hline \multirow{2}{*}{ Ventilator } & \multicolumn{3}{c}{ Absolute Change After Starting Nebulization } \\
\cline { 2 - 5 } & $\begin{array}{c}\text { Inspiratory } \\
\text { Flow } \\
(\mathrm{L} / \mathrm{min})\end{array}$ & $\begin{array}{c}\text { Duration of } \\
\text { Insufflation } \\
(\mathrm{ms})\end{array}$ & $\begin{array}{c}\text { Tidal } \\
\text { Volume } \\
(\mathrm{mL})\end{array}$ & $\begin{array}{c}\text { Peak Airway } \\
\text { Pressure } \\
\left(\mathrm{cm} \mathrm{H} \mathrm{H}_{2} \mathrm{O}\right)\end{array}$ \\
\hline Evita XL & $-1.8 \pm 0.4$ & $20 \pm 4$ & $-10 \pm 7$ & $-0.3 \pm 0.1$ \\
Avea & $-0.1 \pm 0.1$ & $7 \pm 3$ & $7 \pm 4$ & $0.03 \pm 0.06$ \\
Galileo & $0.1 \pm 0.6$ & $14 \pm 4$ & $11 \pm 7$ & $0.3 \pm 0.4$ \\
G5 & $-0.5 \pm 0.3$ & $7 \pm 2$ & $1 \pm 8$ & $-0.2 \pm 0.1$
\end{tabular}

Values indicated were measured at the Y-piece (flow meter 2 on Fig. 1) and are presented as mean $\pm \mathrm{SD}$. All measured changes were statistically significant $(P<.05)$ but clinically negligible.

and desorbed overnight in $50 \mathrm{~mL}$ of $2 \%$ total ionic strength adjustment buffer (Reagecon, Shannon, Ireland). The sodium fluoride extracted was then assayed by electrochemical analysis (Orion combination fluoride electrode, Thermo Fisher Scientific, Waltham, Massachusetts) and expressed as a percentage of the total amount recovered on both filters. Measurements (triplicate) were made with the Evita $\mathrm{XL}$ ventilator in situations of poor synchrony (short inspiratory time of $0.6 \mathrm{~s}$ ) and in situations of improved synchrony (long inspiratory time and/or reducing the volume of compressible gas proximal to the nebulizer), with the nebulizer being placed close to the Y-piece (Fig. 1). Finally, deposition measurements were also performed, placing the nebulizer in a position $25 \mathrm{~cm}$ proximal in the inspiratory limb. 


\section{Ventilator-Integrated Jet Nebulization Systems}

A

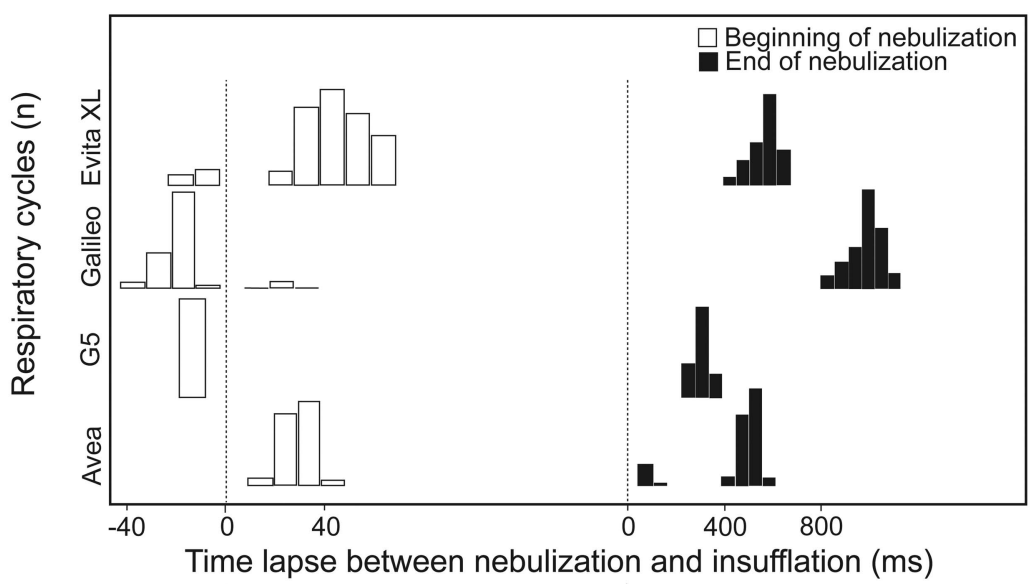

B

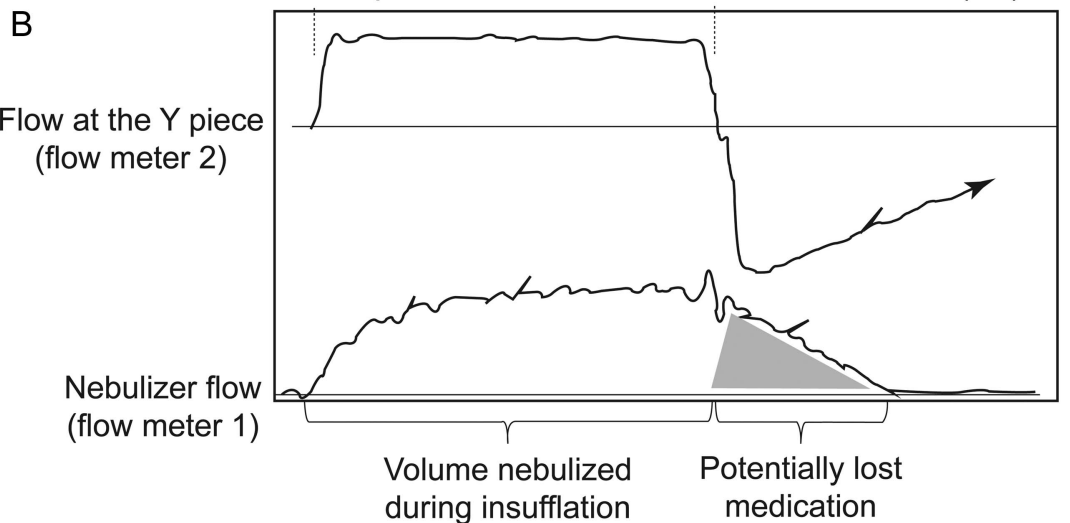

Fig. 2. Synchronization of nebulization and insufflation. A: synchronization at the beginning and end of insufflation is depicted. Bars represent number of respiratory cycles exhibiting the given time lapse between nebulization and insufflation, thus representing the distribution of time lapse for the 180 ventilator cycles analyzed for each ventilator (20 cycles analyzed per ventilator setting: tidal volume 300 , 500 , and $700 \mathrm{~mL}$ and inspiratory flow 30,50 , and $70 \mathrm{~L} / \mathrm{min}$ ). Of note, in some cases, nebulization seems to start a few milliseconds before insufflation; this may be due to small artifacts and measurement precision. B: Typical ventilator and nebulizer flow tracings as observed for all tested ventilators. At the beginning of insufflation, one observes a rapid start of nebulization; however, maximum nebulization flow is observed only later during insufflation. At the end of insufflation, nebulization flow starts decreasing; however, as nebulization persists, some potential loss of medication may occur.

Measurements were reported as mean $\pm \mathrm{SD}$. For each ventilator, comparisons were carried out using the Student $t$ test and the Pearson correlation coefficient. Comparisons between ventilators were made using analysis of variances, followed, if significant, by two-by-two post hoc analysis. A $P$ value below .05 was considered significant.

\section{Results}

Altogether, 2,880 respiratory cycles were analyzed.

\section{Control of $\mathbf{V}_{T}$}

When starting the nebulizer system, the change in $\mathrm{V}_{\mathrm{T}}$ was always below $20 \mathrm{~mL}$ in absolute value and below $10 \%$ of the set $\mathrm{V}_{\mathrm{T}}$. Minimal changes observed in inspiratory flow and in the resulting $\mathrm{V}_{\mathrm{T}}$ and airway pressure, as well as the gas flow passing through the nebulizer, are detailed in Table 1.

\section{Inspiratory Synchronization}

Typical nebulizer and ventilator flow waveforms are depicted in Figure 2. At the beginning of insufflation, the time lapse between the start of insufflation and that of nebulization was always below $100 \mathrm{~ms}$. Following this rapid start, nebulization flow gradually increased to a maximum during insufflation. At the end of insufflation, nebulization flow gradually decreased to zero. Due to this gradual decrease, prolonged nebulization persisted after the end of insufflation, until up to $1 \mathrm{~s}$ (Fig. 2 and Table 2). For all ventilators, this persisting expiratory nebulization (poor end-inspiratory synchronization) explained that up to $80 \%$ of nebulization could occur and be potentially lost during expiration. This proportion was negatively correlated with the insufflation time set on the ventilator $\left(r^{2}=0.45, P<.001\right)$ (Fig. 3).

For the Evita $\mathrm{XL}$ ventilator, results concerning $\mathrm{V}_{\mathrm{T}}$ control and synchronization were similar when using nebu- 


\section{Ventilator-Integrated Jet Nebulization Systems}

Table 2. Synchronization and Aerosol Produced During Expiration

\begin{tabular}{lccc}
\hline \hline Ventilator & $\begin{array}{c}\text { Beginning of Insufflation Synchrony } \\
\text { (Time Lapse Between Beginning of } \\
\text { Insufflation and of Nebulization, ms)* }\end{array}$ & $\begin{array}{c}\text { End-Insufflation Synchrony } \\
\text { (Time Lapse Between End of } \\
\text { Insufflation and of Nebulization, ms) } \dagger\end{array}$ & $\begin{array}{c}\text { Potential Loss of Medication } \\
\text { (Proportion of Volume Nebulized } \\
\text { During Expiration, \%) }\end{array}$ \\
\hline Evita XL & $40 \pm 18(-15,70)$ & $550 \pm 48(445,625)$ & $38 \pm 13(17,63)$ \\
Avea & $30 \pm 5(20,45)$ & $460 \pm 130(80,575)$ & $24 \pm 11(0,43)$ \\
Galileo & $20 \pm 9(-45,30)$ & $970 \pm 60(815,1,095)$ & $49 \pm 12(27,75)$ \\
G5 & $-10 \pm 2(-15,-10)$ & $310 \pm 26(245,375)$ & $19 \pm 6(10,31)$
\end{tabular}

Values are indicated as mean \pm SD (range). All differences were statistically significant (overall analysis of variance and two-by-two post hoc comparisons). Only the slightly better performance of the G5 ventilator may be clinically relevant. For the Evita XL ventilator, results were similar when using nebulizer 8412935 as recommended (data not shown).

* Represented as $\Delta 1$ in Figure 1.

$\dagger$ Represented as $\Delta 2$ in Figure 1.

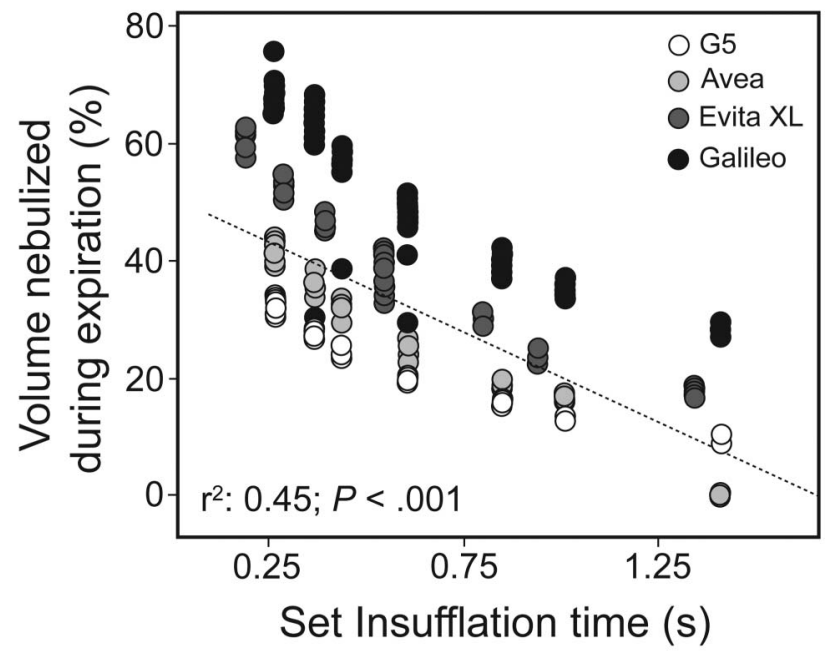

Fig. 3. The gas volume passing through the nebulizer during expiration (expressed as a percentage of the total gas volume passing through the nebulizer over the respiratory cycle) potentially generates aerosol during expiration. Volume was measured through integration of the flow signal over the time of inspiration and expiration. This aerosol is believed to be cleared through the expiratory limb and lost for the patient as opposed to aerosol generated during insufflation, which is potentially delivered to the patient. Increasing the set inspiratory time decreases the amount of medication nebulized during expiration, thus potentially increasing the amount of medication delivered to patients.

lizer 8412935 as recommended by the manufacturer; for all ventilators, synchrony was similar whatever the position of the nebulizer in the circuit, in particular placing the nebulizer $25 \mathrm{~cm}$ upstream in the inspiratory limb (data not shown).

\section{Mechanism of Poor Synchronization}

When evaluating synchronization without a nebulizer placed in the circuit (very low resistance opposed to the nebulization port gas flow), an excellent synchronization was observed at the beginning and end of insufflation for all ventilators, with time lapses below $60 \mathrm{~ms}$ whatever the ventilator setting. Conversely, using the high internal resistance nebulizer $\left(12.7 \pm 0.3 \mathrm{~cm} \mathrm{H}_{2} \mathrm{O} / \mathrm{L} / \mathrm{s}\right.$ measured with an air flow of $2 \mathrm{~L} / \mathrm{min}$ ), an extremely large delay between end of insufflation and end of nebulization, up to $2.5 \mathrm{~s}$, was observed for all ventilators. When using the nebulizer with a standard internal resistance $\left(0.67 \pm 0.07 \mathrm{~cm} \mathrm{H}_{2} \mathrm{O} /\right.$ $\mathrm{L} / \mathrm{s}$ ), used for the rest of the experiments, or when reducing the tubing volume of the nebulizer gas supply (reducing the length of the tubing to $2 \mathrm{~cm}$ ), end-insufflation synchronization improved for all ventilators. Those mechanisms of asynchrony are depicted in Figure 4.

\section{Effect of an End-Inspiratory Pause}

The Evita XL and Avea systems delivered nebulization synchronized with insufflation, whereas the Galileo and G5 systems were synchronized with inspiration (active nebulization persisting during the pause) (Fig. 5).

\section{Impact of Poor Synchronization on Aerosol Loss and Delivery}

When the nebulizer was placed close to the Y-piece (Fig. 1), ventilator settings associated with prolonged flow through the nebulizer during expiration (short inspiratory time related to high inspiratory flow and/or reduced $\mathrm{V}_{\mathrm{T}}$ ) were associated with $80 \%$ aerosol deposition on the expiratory limb filter (lost aerosol produced by a sufficient flow through the nebulizer during expiration) and 20\% at the tip of the endotracheal tube (Table 3). When improving inspiratory synchronization, through increasing inspiratory time to $1.40 \mathrm{~s}$ (reduced inspiratory flow and/or increased $\mathrm{V}_{\mathrm{T}}$ ) or reducing the volume of compressible gas proximal to the nebulizer, only $40 \%$ and $47 \%$ of deposition, respectively, occurred on the expiratory limb filter $(60 \%$ and $53 \%$ deposition on the filter placed at the tip of the endotracheal tube). Thus, when placing the nebulizer close to the Y-piece, changes in nebulization during expiration par- 


\section{Ventilator-InTEgrated Jet Nebulization Systems}

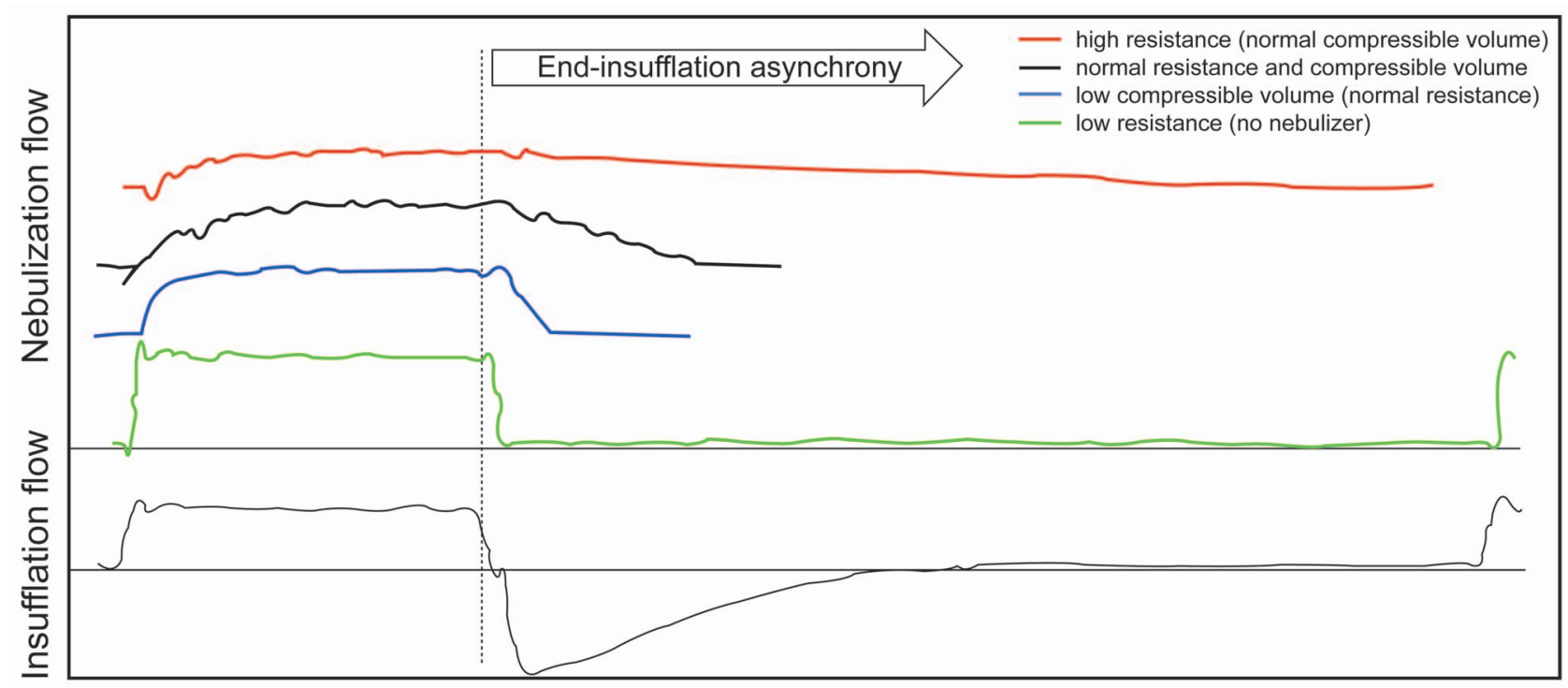

Time

Fig. 4. From top to bottom, nebulizer flow waveforms depict situations with decreasing gas compression/decompression phenomena proximal to the nebulizer. Without a nebulizer, synchronization appeared good (in green); thus, the ventilator per se seemed to deliver gas to the nebulization port in an appropriate manner. However, increasing the internal resistance of the nebulizer (red) or the volume of compressible gas proximal to it (blue versus black curve), situations in which gas compression/decompression proximal to the nebulizer is favored, resulted in long-lasting nebulization during expiration (poor synchronization).

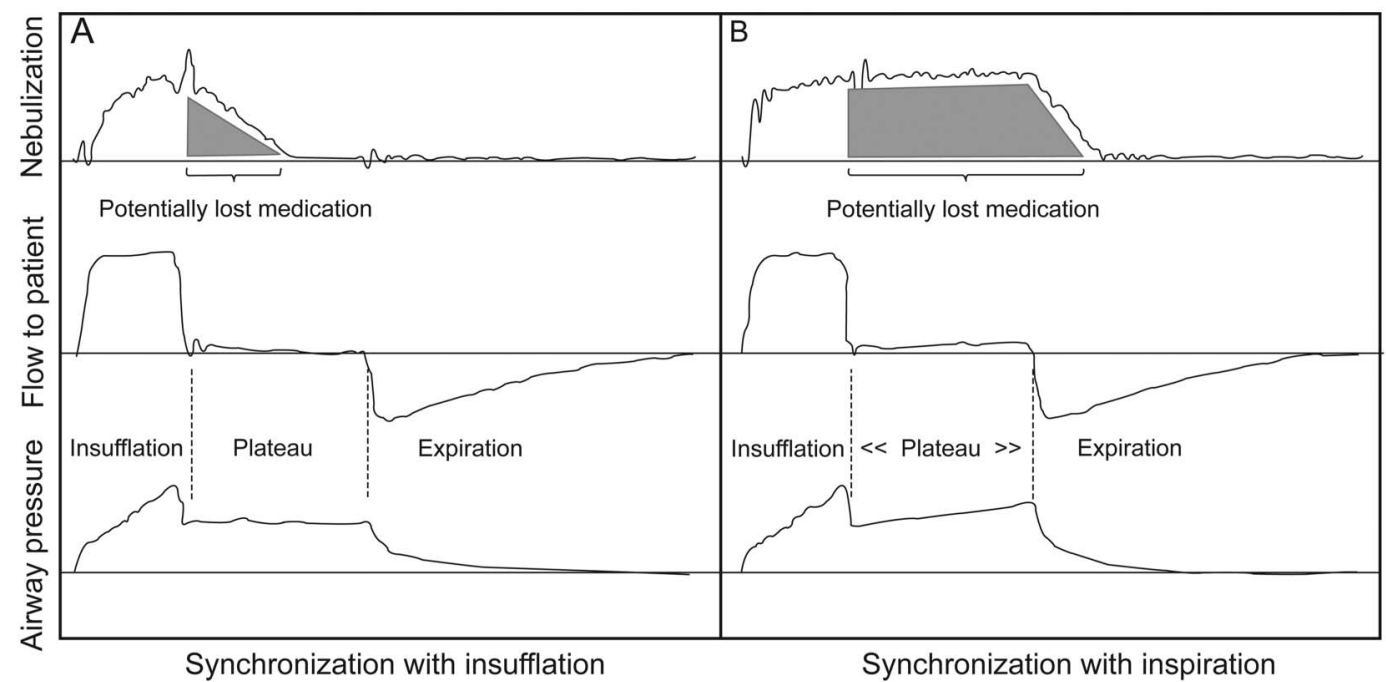

Fig. 5. End-inspiratory pause. A: Depicts behavior of the Evita XL and Avea ventilators when a 1-s end-inspiratory pause was added to the ventilator settings. Nebulization extends within the plateau because of poor end-insufflation synchrony (gas decompression proximal to the nebulizer). B: Depicts behavior of systems synchronized on inspiration rather than insufflation (Galileo and G5 ventilators). Active nebulization persists during the entire end-inspiratory pause, thus increasing airway pressure.

alleled changes in the amount of aerosol lost in the expiratory limb.

Placing the nebulizer upstream in the inspiratory limb was associated with the greatest reduction in deposition on the expiratory limb filter whatever the synchronization and the degree of persistent nebulization during expiration (Table 3).

\section{Discussion}

The main finding of this experimental study is that the $\mathrm{V}_{\mathrm{T}}$ delivered by modern ICU ventilators remains well controlled when integrated jet nebulization systems are used. The synchronization with insufflation was poor, however, with a significant part of the nebulization occurring during 


\section{Ventilator-Integrated Jet Nebulization Systems}

Table 3. Relation Between Persistent Nebulization During Expiration and Aerosol Loss

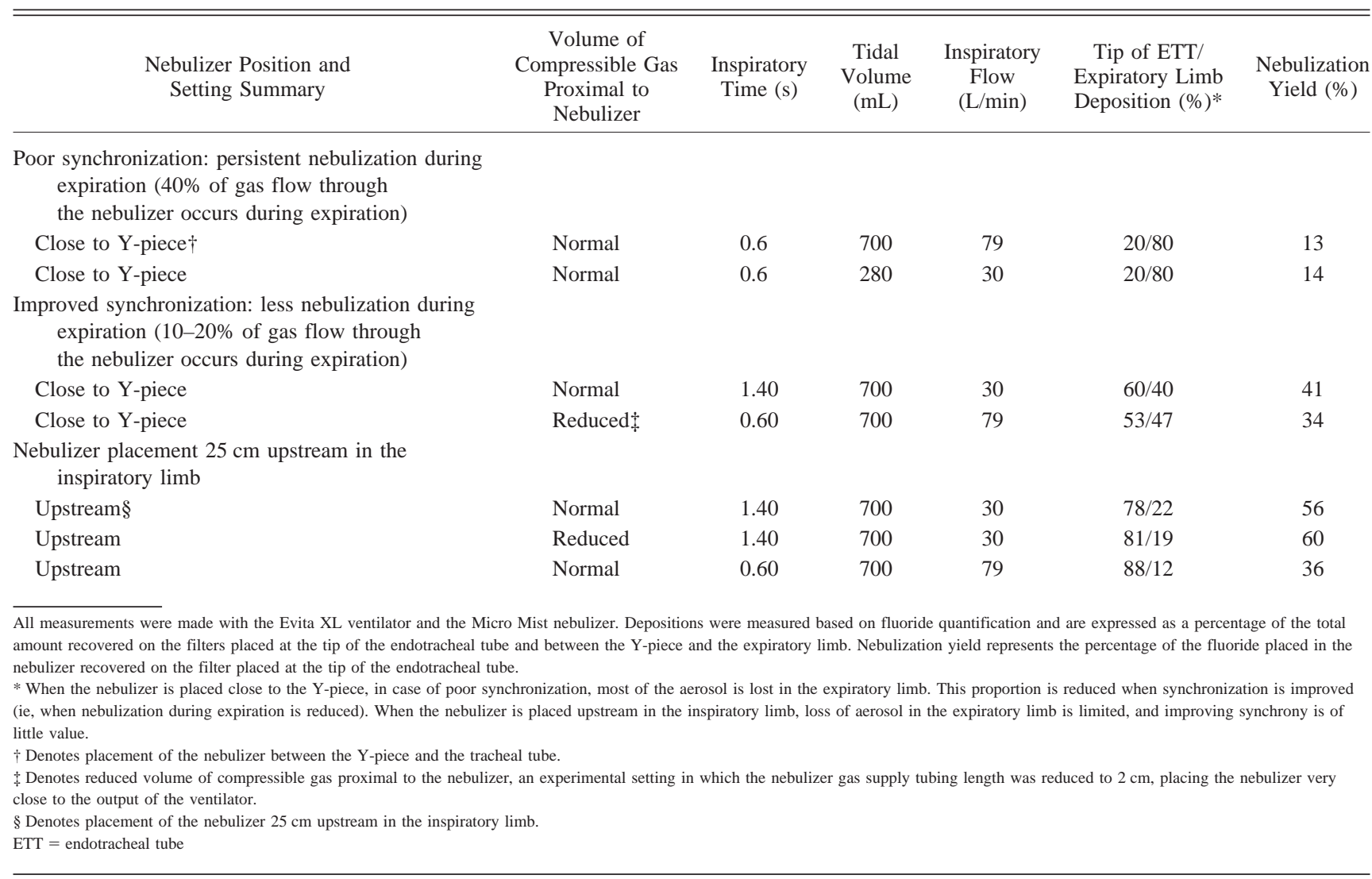

the end-inspiratory pause and/or expiration. This aerosol generated during expiration may be lost if the nebulizer is placed close to the Y-piece. Given the frequent use of those systems, several implications concerning mechanical ventilation settings, nebulization performance, system design, and clinical use might be taken into consideration.

\section{Nebulization Interaction With Ventilation}

When using jet nebulizers during mechanical ventilation, a gas source external to the ventilator induces a significant increase in $\mathrm{V}_{\mathrm{T}}$ as $6-8 \mathrm{~L} / \mathrm{min}$ gas are added to the inspiratory flow. In the present bench study, the change in $\mathrm{V}_{\mathrm{T}}$ observed when activating the integrated systems was always below $10 \%$ of the set $\mathrm{V}_{\mathrm{T}}$, that is, within the precision range of the $\mathrm{V}_{\mathrm{T}}$ setting of modern ICU ventilators. ${ }^{10}$ Thus, those systems may be considered reliable in this regard during volume controlled mechanical ventilation. Previous work evaluating jet nebulization systems integrated in a previous generation of ventilators found that some ventilators delivered a low pressure and a low flow of gas to the nebulizer. ${ }^{5}$ A flow lower than $4 \mathrm{~L} / \mathrm{min}$ has been linked to poor nebulization performance (in terms of nebulization rate and overall yield). For all ventilators, we observed flows through the nebulizer exceeding $6 \mathrm{~L} / \mathrm{min}$ (Table 1). Thus, insufficient gas supply may no longer be a concern or potential factor for improvement in modern ventilators.

\section{Nebulization During Expiration and End-Inspiratory Pause}

Significant nebulization occurred during expiration in all the tested ventilators and may therefore constitute a factor for potential improvement. When the nebulizer is placed close to the Y-piece, medication nebulized during expiration is cleared mostly through the expiratory limb of the ventilator and thus is lost for the patient and has the potential to damage the expiratory flow meter and/or obstruct filters protecting this flow meter. ${ }^{11,12}$ To deliver a higher amount of drug to the patient, it may be desirable to reduce the drug loss during expiration. ${ }^{13,14}$

The Galileo and G5 ventilators are designed to synchronize nebulization with inspiration rather than with insufflation (Fig. 5). No standardization exists, and the value of such a design appears questionable, as medication nebulized during the pause is unlikely to reach the patient. The persistent nebulization during the pause also compromises 


\section{Ventilator-Integrated Jet Nebulization Systems}

proper plateau pressure measurement. The end-inspiratory pause used in clinical practice $(300-500 \mathrm{~ms})$ is usually shorter than the one used for illustrative purposes in this bench study (1 s). Because the flow through the nebulizer is $\sim 4-10$ times lower than the inspiratory flow commonly set on ventilators $(\approx 6-10$ vs $40-60 \mathrm{~L} / \mathrm{min})$, the clinical impact of persistent nebulization during the plateau will be smaller than in our study.

\section{Mechanisms of Asynchrony and Implication for System Design}

The flow coming out of the nebulizer after insufflation has stopped was explained by the gas compressed proximal to the nebulizer. Indeed, testing the integrated nebulization system working without a nebulizer and with a reduced volume of compressible gas proximal to the nebulizer both improved synchronization. By contrast, using a nebulizer with high internal resistance resulted in poor synchronization. Therefore, gas compression and decompression proximal to the nebulizer are probably major determinants of persistent nebulization during expiration.

Synchronization Algorithm. Albeit the ventilators per se did not appear to be at the origin of the asynchrony, a simple way to limit nebulization during expiration would be to stop delivering gas to the nebulizer earlier during inspiration. Actually nebulizing only during the very initial phase of inspiration would limit the amount of medication potentially lost during expiration while also increasing the amount of medication delivered to the distal parts of the lung. Indeed, the gas and thus the aerosol delivered at the end of insufflation do not reach the deep part of the lungs. ${ }^{7,15}$ An ideal algorithm would start nebulization immediately before the start of insufflation (this concerns only fully controlled ventilation) for the nebulizer flow to be highest at the beginning of insufflation. As the particle sizes produced by jet nebulizers decrease with increasing nebulizer flow, such an algorithm would allow production of the finest droplets at the beginning of inspiration, thus favoring distal lung deposition. ${ }^{16}$

\section{Decrease Compressible Gas Volume Proximal to the}

Nebulizer. Reducing the length of the nebulizer gas supply tubing is not feasible in the clinical setting for very practical reasons: the Y-piece and, as a consequence, the head of the patient would need to be placed within very short distance of the ventilator, which is, at best, very unpractical. Thus, reducing the inner tubing diameter may be an interesting approach to reduce the volume of compressible gas.

\section{Clinical Implications}

Internal Resistance of the Nebulizer. Using high-resistance nebulizers leads to very poor synchronization and should probably be avoided. Conversely, a low-resistance nebulizer could be evaluated. However, the larger droplets produced by such devices may be counterproductive (by favoring impaction in the ventilator circuit). ${ }^{17}$

Nebulizer Position in the Circuit. As inspiratory synchronization appeared poor in the present bench study, nebulizer placement may best be done as for continuous nebulizers, that is, proximal in the inspiratory limb, as this placement was associated with the least loss of medication in the expiratory limb and the highest amount of medication delivered at the tip of the endotracheal tube (Table 3). ${ }^{17}$ Precise optimal placement deserves further investigations since different values of bias flow may be associated with different optimal positions. Of note, similar results were observed previously with synchronized jet nebulization systems by O'Doherty et al ${ }^{18}$ and Hughes and Saez. ${ }^{19}$ Beyond confirming those results on modern ventilators, our results show that the benefit of positioning these synchronized systems upstream in the inspiratory limb is due to the observed persistent nebulization during expiration, that is, the nebulizer behaves like a continuous system due to poor synchronization. Thus, our results show that nebulizer placement close to the Y-piece, a practice acknowledged by a large number of intensivists, ${ }^{3}$ is far from optimal for the synchronized jet nebulization systems evaluated in the present study. Whether this placement is appropriate for other devices, potentially better synchronized with inspiration, deserves further evaluation. ${ }^{15}$

Inspiratory Time. As placement of the nebulizer upstream in the inspiratory limb may be difficult in clinical practice, especially with disposable circuits (need for extra tubing and connection), a simple way to reduce the amount of medication potentially lost during expiration when using the integrated jet nebulization systems placed close to the Y-piece may be to increase the inspiratory time (increase in $\mathrm{V}_{\mathrm{T}}$ and/or decrease in inspiratory flow according to the patient's condition and tolerance). Of note, reducing inspiratory flow may be particularly interesting, as it may increase nebulization yield through other means (reduced droplet impaction in the ventilator circuit). ${ }^{16}$ However, placement upstream in the inspiratory limb had the greatest impact on delivery in the systems tested in the present study (Table 3).

\section{Study Limitations}

The results of the present bench study need to be confirmed in patients receiving aerosolized medication. How- 


\section{Ventilator-Integrated Jet Nebulization Systems}

ever, the good results observed concerning $\mathrm{V}_{\mathrm{T}}$ control are unlikely to be very different in the clinical setting, as during volume controlled ventilation, the $\mathrm{V}_{\mathrm{T}}$ is independent of the patient. Pressure-regulated modes of mechanical ventilation were not evaluated in the present bench study; thus, no conclusion may be drawn concerning the function of the integrated jet nebulization systems in this situation. Of note, as $\mathrm{V}_{\mathrm{T}}$ partly depends on the patient's effort and respiratory mechanics, its control by the ventilator would be less relevant. Nebulization is unlikely to affect the delivered pressure, as this variable is very tightly controlled by modern inspiratory valves.

The different test conditions influencing expiratory nebulization also impacted nebulization during inspiration. Our findings strongly suggest that persistent nebulization during expiration is an important mechanism of drug loss. An associated change in nebulization characteristics during inspiration (mainly higher or lower flow through the nebulizer) cannot, however, be completely ruled out. Some variables such as the triggering type, breathing frequency, and pressure in the connecting tubing were not fully evaluated in the present study, although they may interact with some results.

Finally, only $0.9 \%$ sodium chloride/fluoride was used for nebulization in the present study. Behavior may be slightly different with other medications, particularly in the case of solutions exhibiting high viscosity.

\section{Conclusions}

Jet nebulization systems integrated in modern ICU ventilators allow a good control of $\mathrm{V}_{\mathrm{T}}$ and thus may be preferred to the use of external gas sources and empirical ventilator setting changes. Inspiratory synchronization sometimes appeared poor, due to persistent nebulization during the inspiratory pause and expiration, in part because of gas compression/decompression proximal to the nebulizer. Specific settings such as increasing inspiratory time might help to optimize the dose of drug delivered. Importantly, nebulizer placement upstream in the inspiratory limb is the most powerful means of reducing the loss of medication in the expiratory limb due to persistent nebulization during expiration. Delivering nebulization during the entire inspiration, that is, including the pause, appears questionable.

\section{REFERENCES}

1. Dolovich MB, Dhand R. Aerosol drug delivery: developments in device design and clinical use. Lancet 2011;377(9770):1032-1045.
2. Craven DE, Lichtenberg DA, Goularte TA, Make BJ, McCabe WR. Contaminated medication nebulizers in mechanical ventilator circuits: source of bacterial aerosols. Am J Med 1984;77(5):834-838.

3. Ehrmann S, Roche-Campo F, Sferrazza Papa GF, Isabey D, Brochard L, Apiou-Sbirlea G. Aerosol therapy during mechanical ventilation: an international survey. Intensive Care Med 2013;39(6): 1048-1056.

4. Dhand R. Aerosol delivery during mechanical ventilation: from basic techniques to new devices. J Aerosol Med Pulm Drug Deliv 2008; 21(1):45-60.

5. McPeck M, O'Riordan TG, Smaldone GC. Choice of mechanical ventilator: influence of nebulizer performance. Respir Care 1993; 38(8):887-895

6. Miller DD, Amin MM, Palmer LB, Shah AR, Smaldone GC. Aerosol delivery and modern mechanical ventilation: in vitro/in vivo evaluation. Am J Respir Crit Care Med 2003;168(10):1205-1209.

7. Dhand R, Sohal H. Pulmonary drug delivery system for inhalation therapy in mechanically ventilated patients. Expert Rev Med Devices 2008;5(1):9-18.

8. Ehrmann S, Mercier E, Vecellio L, Ternant D, Paintaud G, Dequin PF. Pharmacokinetics of high-dose nebulized amikacin in mechanically ventilated healthy subjects. Intensive Care Med 2008;34(4): 755-762.

9. Dennis JH, Stenton SC, Beach JR, Avery AJ, Walters EH, Hendrick DJ. Jet and ultrasonic nebulizer output: use of a new method for direct measurement of aerosol output. Thorax 1990;45(10):728-732.

10. Lyazidi A, Thille AW, Carteaux G, Galia F, Brochard L, Richard JC. Bench test evaluation of volume delivered by modern ICU ventilators during volume-controlled ventilation. Intensive Care Med 2010; 36(12):2074-2080.

11. Mojoli F, Iotti GA, Imberti R, Braschi A. The importance of protecting the mechanical ventilator during colistin methanesulfonate nebulization. Intensive Care Med 2013;39(3):535-536.

12. Tonnelier A, Lellouche F, Bouchard PA, L'Her E. Impact of humidification and nebulization during expiratory limb protection: an experimental bench study. Respir Care 2013;58(8):1315-1322.

13. Athanassa ZE, Markantonis SL, Fousteri MZ, Myrianthefs PM, Boutzouka EG, Tsakris A, Baltopoulos GJ. Pharmacokinetics of inhaled colistimethate sodium (CMS) in mechanically ventilated critically ill patients. Intensive Care Med 2012;38(11):1779-1786.

14. Lu Q, Luo R, Bodin L, Yang J, Zahr N, Aubry A, et al. Efficacy of high-dose nebulized colistin in ventilator-associated pneumonia caused by multidrug-resistant Pseudomonas aeruginosa and Acinetobacter baumannii. Anesthesiology 2012;117(6):1335-1347.

15. Luyt CE, Clavel M, Guntupalli K, Johannigman J, Kennedy JI, Wood $\mathrm{C}$, et al. Pharmacokinetics and lung delivery of PDDS-aerosolized amikacin (NKTR-061) in intubated and mechanically ventilated patients with nosocomial pneumonia. Crit Care 2009;13(6):R200.

16. Laube BL, Jashnani R, Dalby RN, Zeitlin PL. Targeting aerosol deposition in patients with cystic fibrosis: effects of alterations in particle size and inspiratory flow rate. Chest 2000;118(4):1069-1076.

17. Ari A, Fink JB, Dhand R. Inhalation therapy in patients receiving mechanical ventilation: an update. J Aerosol Med Pulm Drug Deliv 2012;25(6):319-332.

18. O'Doherty MJ, Thomas SH, Page CJ, Treacher DF, Nunan TO. Delivery of a nebulized aerosol to a lung model during mechanical ventilation. Am Rev Respir Dis 1992;146(2):383-388.

19. Hughes JM, Saez J. Effects of nebulizer mode and position in a mechanical ventilator circuit on dose efficiency. Respir Care 1987; 32(12):1131-1135. 